\title{
Transform Domain Based Iris Recognition using EMD and FFT
}

\author{
Sateesh Kumar $\mathrm{H} \mathrm{C}^{1}$, Abhilash $\mathrm{S} \mathrm{K}^{2}$ and Raja $\mathrm{K} \mathrm{B}^{2}$ and Venugopal K R ${ }^{2}$ \\ ${ }^{I}$ Department of Electronics and Communication Engg, Sai Vidya Institute of Technology, Bangalore, India \\ ${ }^{2}$ University Visvesvaraya College of Engineering, Bangalore University, Bangalore, India
}

\begin{abstract}
Iris is one of the physiological trait which is used to identify the individuals. In this paper Transform Domain Based Iris Recognition using EMD and FFT is proposed. Circular Hough Transform is used in the Preprocessing stage to extract circular part of eye. The circular iris part is converted into rectangular rubber sheet model in Region of Interest (ROI).Empirical Mode Functions (EMF)'s are obtained by applying Empirical Mode Decomposition (EMD) on the Iris. FFT is also applied on ROI to extract the features. These features are added arithmetically to obtain final features. The features of the database are compared with test iris using Euclidian Distance $(E D)$ to compute performance parameters. It is observed that the values of CRR and EER are better in the case of propsed algorithm compared to existing algorithms.
\end{abstract}

Keywords: Iris Recognition, EMD, FFT, ROI, ED and Fusion

\section{Introduction}

Biometrics is used to authenticate a person based on physiological and behavioral characteristics of human beings. The physiological traits are Face, Fingerprint, Palm print, DNA and Iris of a person is constant in nature throughout their lifetime. The behavioral traits are Signature, Voice, Keystroke and Gait of a person are based on mood, age and surrounding circumstances, hence are not constant in their life time. The iris is considered for our research work as it is unique, non-invasive features to recognize human beings properly. Iris is a circular part of an eye and lies between sclera and pupil. The iris is an internal organ of human and is well protected compared to other physiological traits. The disadvantage of an iris is that the person has to co-operate to acquire iris image and also the iris image cannot be acquired from long distances. The application of iris recognition system is widely used in airport checking, refugee control, military applications, homeland security, various private and public sectors and the national identification AADHAR systems.

Contribution- In this paper IRFEF algorithm is proposed. The ROI area is extracted from iris using preprocessing. The EMD and FFT is then applied on ROI to generate individual feature vector. The final feature vectors are obtained by fusing individual features. The ED is used to compare test image features with the database image features.

Organization- The paper is organized as section 1 is introduction. The related literature survey is discussed in section 2.The proposed model is described in section 3. The algorithm is presented in section 4. The performance analysis and conclusions are given in section 5 and section 6 respectively.

\section{Literature Survey}

The application and usage of Iris as a biometric characteristic for individual identification with different technique are described in this section.Ya-Ping Huang et al., [1] proposed an Iris Recognition System which adopts Independent Component Analysis (ICA) in which the iris pattern is represented by ICA coefficients. It determines the centre of each class by competitive learning mechanism to recognize the iris pattern. Tisse et al., [2] proposed a technique which is based on the extraction of instantaneous features in the Iris texture which are the emergent frequency and/or instantaneous phase It is an alternate solution to Daugman's mathematical algorithms for local feature extraction, which allows adjusting the size of the biometric signature without increasing the computation complexity. Daugman [3] is implemented integrodifferential operators to detect the inner and outer Iris boundaries. 2-D Gabor filters are applied to extract unique binary vectors constituting an Iris features. Daugman used a statistical matcher (logical exclusive OR operator) which computes the average Hamming Distance between two Iris codes. Hui Zheng and Fei Su [4] proposed an iris recognition using Gabor wavelet method; it includes the iris localization, eyelids detection and the optical filter parameters selection. Lahouari Ghouti and Fares S.Al-Qunaieer [5] proposed Quaternion Phase Correlation for color iris recognition based on a new hyper complex phase-based color iris recognition and matching method is used which has greater accuracy, flexibility in capturing the color iris information and reduces the complexity. Ghassan J. Mohammad and hong BinKung et al., [6] proposed an efficient iris localization algorithm based on Angular Integral Projection Function method The algorithm adopts boundary point detection along with curve fitting and it does not require to find all the boundary points so the localization 
speed will be very fast, two sets of radial boundaries points for the iris inner and outer boundaries are obtained by fitting a circle for each of the points. Then 2D Gabor filter is used to extract the iris code for the normalized iris image. Khin Sint Kyaw [7] proposed a straight forward approach for segmenting the iris patterns and an automated global threshold finds pupil centre. The performance of iris recognition system has been enhanced using the statistical features and then comparison of two iris patterns by using Hamming distance which includes the preprocessing system, segmentation, feature extraction and recognition. Kekre et al., [8] proposed an iris recognition system based on vector quantization. The algorithms such as LBG, KPE and KFCG are implemented.KFCG has the best performance with the accuracy of $89.10 \%$ and requires $99.79 \%$ less computations compared to LBG and KPE. Zhou Hu-Lin and Xie Mei [9] proposed iris matching, using repeated executable portion of iris recognition algorithm which is run parallel on FPGA system, which results in 22 times more faster and the power consumption is low compared to earlier sequential digital systems, such as simple DSPs which matches data one by one consuming more time. Patil and Sudharshan Patilkulkarni [10] proposed a wavelet based quality measure for iris images along with appropriate de-noising procedure. It has three modules namely image preprocessing, feature extraction and recognition modules. Two iris images are compared using Euclidian distance. Hong-ying, et al., [11] proposed an iris feature extraction approach using both spatial and frequency domain considering the texture of the iris as a kind of geometrical figure. Steer able pyramid is implemented to get the orientation information on iris images. Non-symmetrical SVM is employed as classifier. Feature sequence combined with spatial and frequency domain represents the variation details of the iris patterns properly. Makram Nabti and Ahmed Bouridane [12] proposed an efficient multi-scale approach for human iris recognition, based on combined feature extraction methods by taking both the textural and topological features of iris image. Multiscale edge detection method is used for localization of iris and a feature extraction technique used is based on the combination of multiscale feature extraction techniques, the combined multi resolution feature extraction technique gives a better performance with respect to accuracy.

\section{Proposed Model}

In this section, definitions and proposed model are discussed.

\subsection{Definitons}

(i) False Accept Rate or False Match Rate (FAR or FMR): The probability that the system incorrectly matches the wrong person from out of database. It is the ratio of number of person accepted to the total number of persons out of database, given as an Equation 1.

\section{FAR Number of persons accepted from out of database \\ Totalnumber of persons in the out of database}

(ii)False Rejection Rate or False Non-Match Rate (FRR or FNMR): The probability that the system fails to detects a match correct person in the database. It is the ratio of number of correct persons rejected in database to the total number of persons in database is given in the Equation 2.

FRFRR $=\frac{\text { Number of correct persons rejected }}{\text { Totalnumber of persons in the database }}$

(iii) Equal Error Rate or Crossover Error Rate (EER or CER): The rates at which both accept and reject errors are equal. The value of the EER can be easily obtained from the FRR and FAR v/s Threshold curve. The EER is a quick way to compare the accuracy of devices. In general; the device with the lowest EER is most accurate.

(iv) Correct Recognition Ratio or True Success Rate (CRR or TSR): The rate at which the system recognizes all the persons in the database as particular individuals correctly. It measures the correctness of the technique used for recognition.CRR is defined as the ratio of number of persons correctly matched to the total no of persons in the database is given in the Equation 3.

$C R R=\frac{\text { Number of persons correctly matched }}{\text { Totalnumber of persons in the database }}$.

3.2 MODEL

The block diagram of Proposed model to identify a person using iris biometric parameter is shown in the Figure1. The eye image is preprocessed to obtain iris part. The features of the iris are extracted using EMD and FFT. The Euclidean Distance is used for comparison between database and test image. 


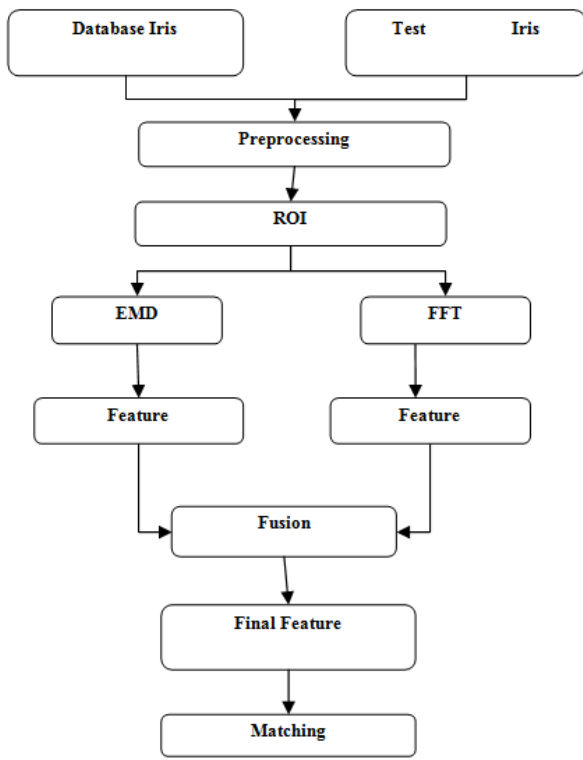

Fig.1: Proposed Iris Recognition Model.

\subsubsection{Iris Database:}

The database from CASIA-IrisV3 is considered and has 108 person's iris images and 7 images per person ie., total number of images are 756.The 70 persons are used to create a database and the remaining 38 persons are considered as out off database. The 6 images per person for 70 person's are used for database i.e., total number of images in the database are 420 and one image per person is considered as test image to compute FRR and CRR. The FAR is computed using out off database i.e., from 38 persons.

\subsubsection{Preprocessing:}

The iris part from an eye image is obtained using preprocessing such as (i) Iris Localization and (ii) Iris Normalization.

\section{i)Iris Localization:}

The iris localization is to detect the iris area between pupil and sclera from an eye image. The canny edge detection technique is allowed for weighting of the gradients and uses a multiple stage algorithm to detect a wide range of edges. Circular Hough Transform (CHT) is used to find patterns of circles in an eye image. CHT is shown in the Figure2. Basically, the detection of circle by CHT involves ease of computation and implementation but takes large computational time and storage space. The iris region can be approximated by two circles, one for the iris/sclera boundary and another interior to the first for the iris/pupil boundary. The eyelids and eyelashes normally occlude the upper and lower parts of the iris region. The initial stage begins with locating the center of the iris and determines the radius of the pupil in order to separate the iris image.

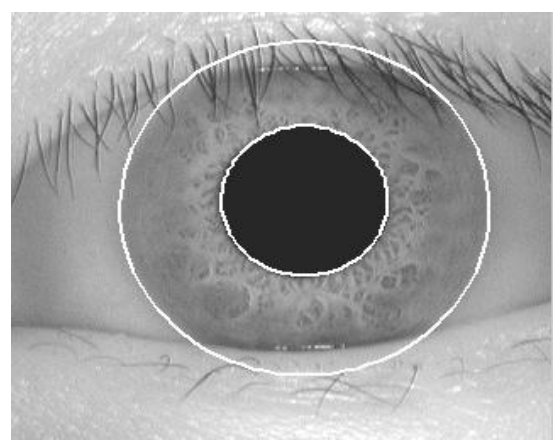

Fig.2: After Hough transform

\section{ii) Iris Normalization:}

The variations in an eye image such as size of iris, position of iris in an eye image, orientation of iris and images acquired at different timings and different distances leads to different patterns of eye images. Hence iris normalization is required to arrange all iris images of a person to similar dimensions. Normalization process involves unwrapping the iris and converting into polar equivalent using Daugman's Rubber sheet model [13]. 
The center of the pupil is considered as the reference point and a remapping formula is used to convert the points on the Cartesian scale to the polar scale using equation 4 and 5 as shown in the Figure 3.

$$
r^{\prime}=\sqrt{\alpha \beta} \mp \sqrt{\alpha \beta^{2}-\alpha-x_{1}^{2}}
$$

Where,

$r^{\prime}=$ represents the distance between the edge of the pupil and edge of the iris at an angle $\theta$ round the region

$r \boldsymbol{l}=$ iris radius

$$
\alpha=0_{x}^{2}+0_{y}^{2}
$$

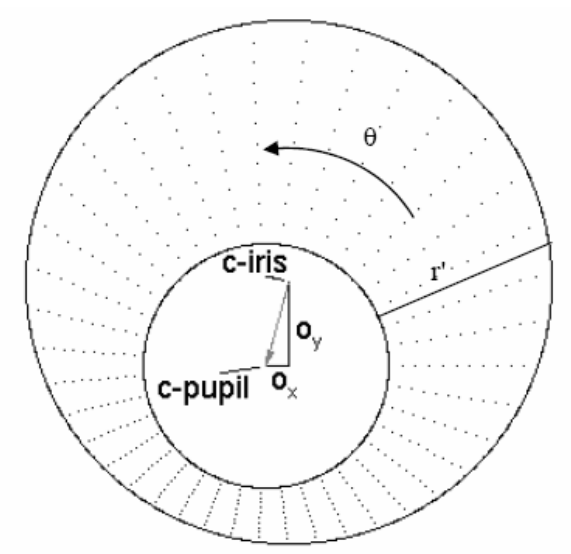

Fig.3: Normalization process

ox,oy=represent the displacement of the centre of the pupil relative to the centre of the iris

$$
\beta=\cos \left(\pi-\arctan \left(\frac{O_{y}}{O_{x}}\right)-\theta\right)
$$

The homogeneous rubber sheet model devised by Daugman remaps each point within the iris region to a pair of polar coordinates where the radius $r$ is on the interval $(0,1)$, and the angle is on the interval $[0,2 \pi]$. Then the normalized iris region is unwrapped into a rectangular region is shown in Figure 4.

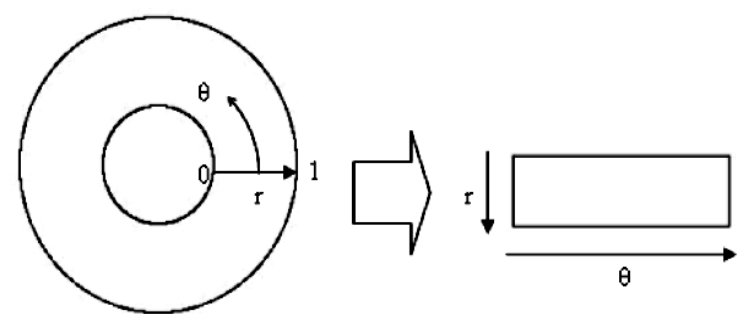

Fig.4: Daugman's Rubber Sheet Model

\subsubsection{Region of Interest (ROI):}

The Region of Interest is one of the major factors which influence the speed and quality of Iris recognition. The Iris portion obtained from pre-processed is of the size $64 X 512$ would be practically heavy to process is shown in Figure 5.

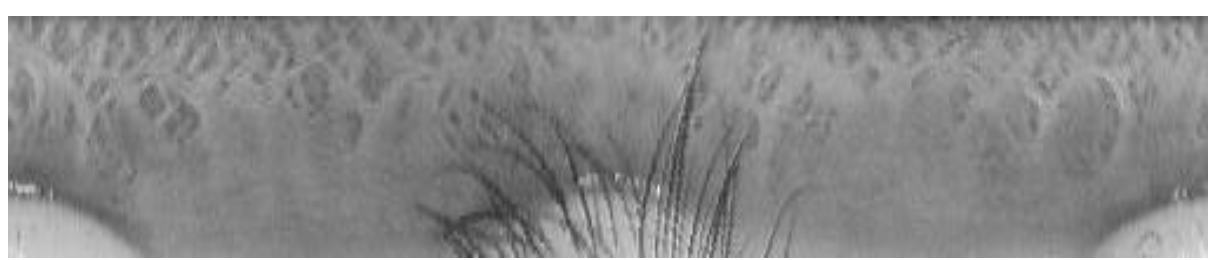

Fig.5: Pre-processed Iris Image.

The small portion from the preprocessed iris with significant information is considered to reduce the recognition error and computation time which leads to ROI. On observation of CASIA database intersection of $6 \%$ to $19 \%$ of horizontal portion and $40 \%$ to $90 \%$ of vertical portion of preprocessed iris is considered for ROI as it consists of significant features to differentiate iris images of two different persons. The extraction process of ROI from iris is shown in Figure 6. The ROI of size $8 * 250$ is shown in the Figure 7 


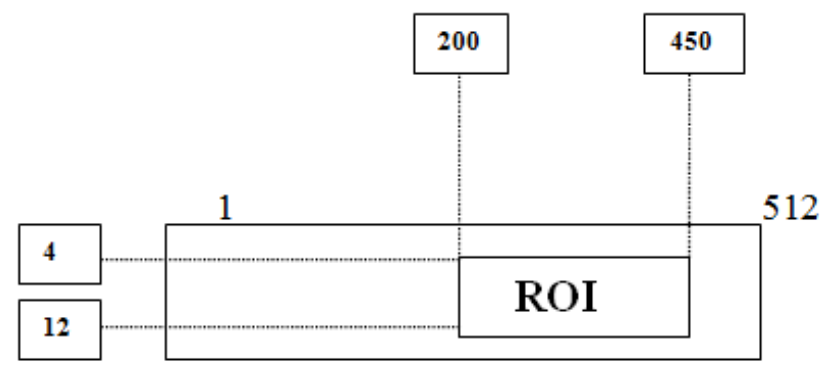

64

Fig.6: ROI Extraction

Fig.7: ROI of size $8 * 250$.

\subsubsection{Empirical Mode Decomposition (EMD):}

The EMD [19] [20] is a multi-resolution decomposition technique and suitable to analyze non-linear and non-stationary signals. EMD can decompose a signal into several Intrinsic Mode Functions (IMFs). The signal component is said to be IMF only if the signal satisfies the two conditions such as (i) In the whole dataset of the signal, the number of extremas and the number of zero-crossings must either be equal or differ at most by one and (ii) At any point, the mean value of the envelope defined by the local maxima and the envelope defined by the local minima must be zero.

Unlike the harmonics function of a Fourier series, these oscillatory functions may vary in both amplitude and frequency over time. In this decomposition process, the first IMF contains the highest frequencies associated with the original signal; each subsequent IMF contains lower frequency components The pixel sequences of ROI image are concatenated by considering row wise to form 1-Dimensional vector given in an Equation 6.

$\mathrm{V}=\left\{\mathrm{v}_{1} ; \mathrm{v}_{2} ; \mathrm{v}_{3} \ldots \mathrm{v}_{\mathrm{n}}\right\}$.

Where $, \mathrm{v}_{1}, \mathrm{v}_{2} \ldots . \mathrm{v}_{\mathrm{n}}$ are rows of ROI

$\mathrm{n}=$ size of ROI $8 * 250$

An EMD is applied on vector V of ROI is shown in Figure 8.
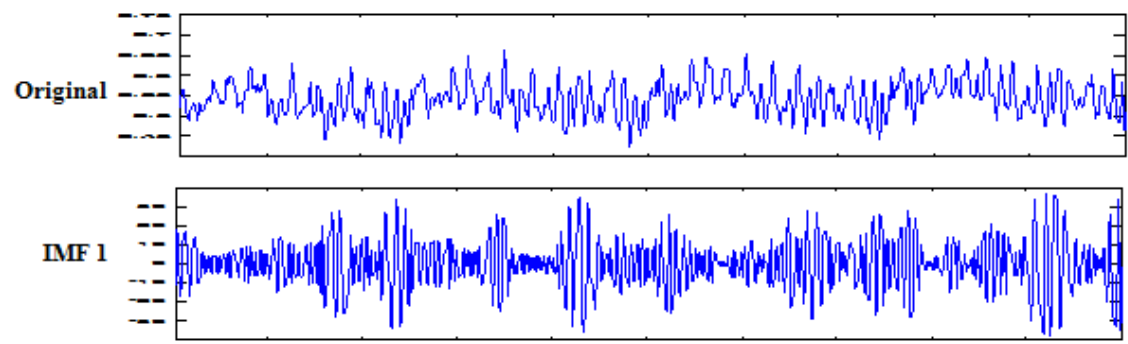

IMF 2
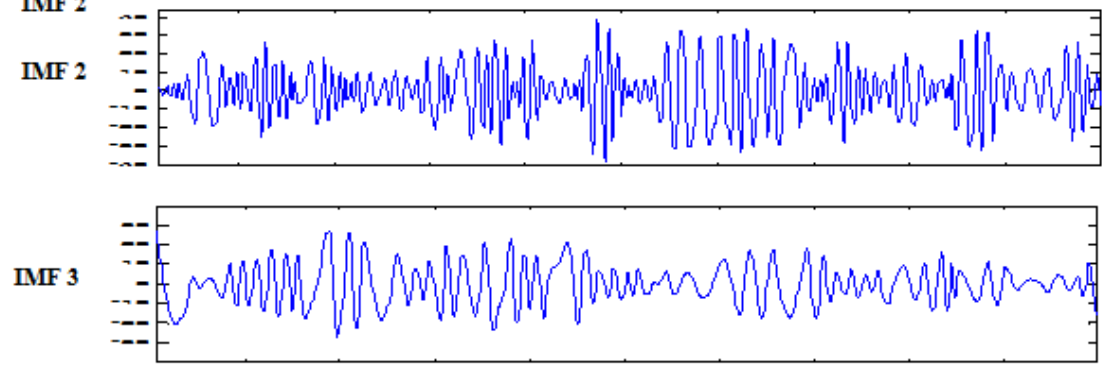

IMF 4

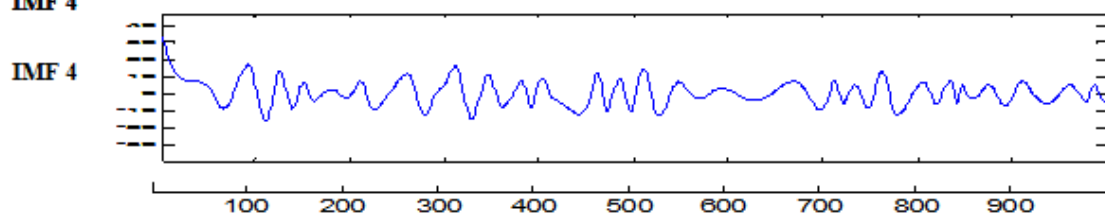

Fig.8: The four IMF components obtained for the original iris signal 
The first IMF is obtained when the signal satisfies the given conditions in the EMD process. Then the first IMF is subtracted from the original signal, and the remaining signal is again checked for the given conditions in the EMD, when the subtracted signal satisfies the given condition. The IMF 2 is obtained similarly the next IMF's are determined. The IMF1 is discarded as it has full of high frequency component which is not suitable to extract feature. The IMF2, IMF3, IMF4 are considered for feature vector and add them to get to new vector.

The features of EMD are formed by an addition [20] of the IMF2, IMF3 and IMF4 as given in an Equation 7.

$\mathrm{EMD}=(\mathrm{IMF} 2+\mathrm{IMF} 3+\mathrm{IMF} 4)$

\subsubsection{Feature Extraction Using FFT}

The FFT is applied to the ROI vector V of one-dimensional and the absolute coefficients are computed and considered as the feature vector. The FFT spectrum of ROI vector is shown in Figure 9.

\subsubsection{Final Feature Set}

The EMD and FFT features are fused element by element using arithmetic summation to generate final feature vector. The fused features of EMD and FFT have better recognition than an individual features. The final features vector is given in an Equation 8.

Vector $=[\mathrm{EMD}+\mathrm{FFT}]$.

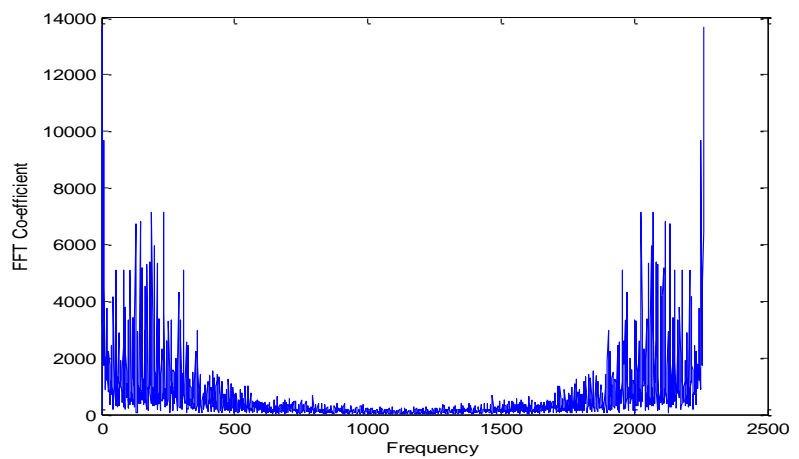

Fig.9: FFT of ROI

\subsubsection{Final Feature Set}

The EMD and FFT features are fused element by element using arithmetic summation to generate final feature vector. The fused features of EMD and FFT have better recognition than an individual features. The final features vector is given in an Equation 8.

Vector $=[\mathrm{EMD}+\mathrm{FFT}]$.

3.2.6 Matching

The final feature vector of test image is compared with final feature vectors of database images using Euclidean distance to verify whether the test image is matching with database images. The Euclidean distance formula is given in the Equation 9.

$d 1(p, q)=\sqrt{\frac{1}{M} \sum_{i=1}^{M}\left(p_{i}-q_{i}\right)^{2}}$.

Where, $\mathrm{M}=1 * 2000$ the dimension of feature vector.

$p_{i}$-is the database feature vector.

$q_{i}$-is the test feature vector.

\section{Algorithm}

Problem definition: The iris is used to identify a person. The EMD and FFT are used to generate features individually. The final features are obtained by fusing individual features. The objectives are

1. Recognize a human using Iris

2. To increase $\mathrm{CRR}$ value

3. To decrease EER value

The correct recognition of a person using iris by fusion of EMD and FFT features are explained in detail in the Table 1 
Table 1: Proposed Algorithm

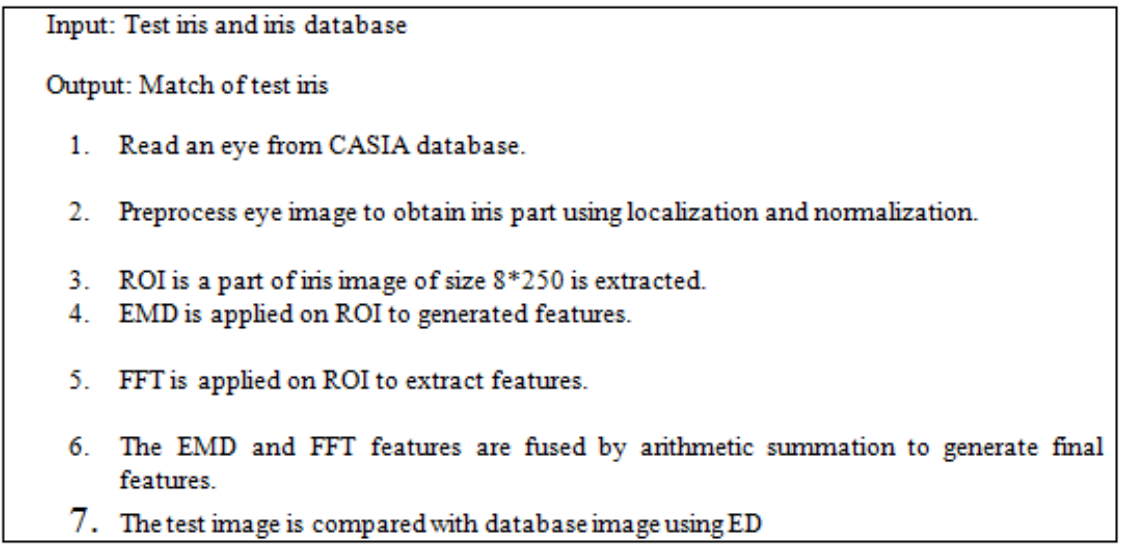

\section{Results}

For performance analysis the CASIA V3 iris database is considered is shown in Figure 10. The FRR value decreases and FAR value increases for the proposed technique with respect to their threshold as shown in Figure 11. The value of FAR, FRR and EER are zero at threshold of 0.3 . Hence our technique is better in terms of FAR, FRR and EER.
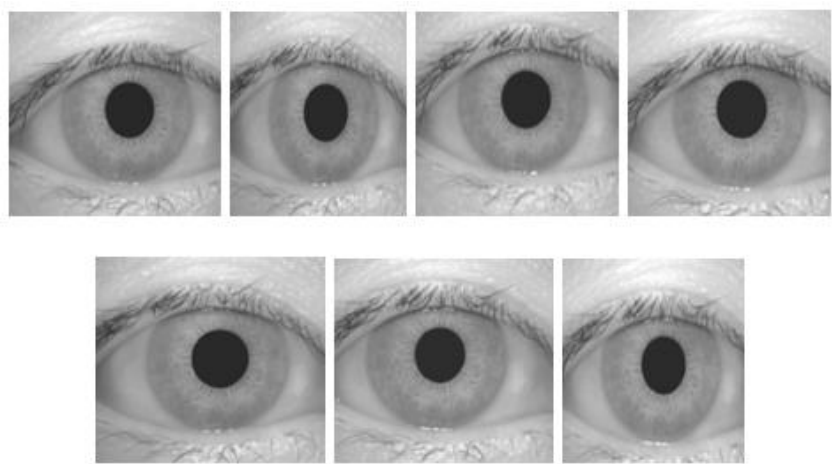

Fig.10: Sample Iris images of CASIA database

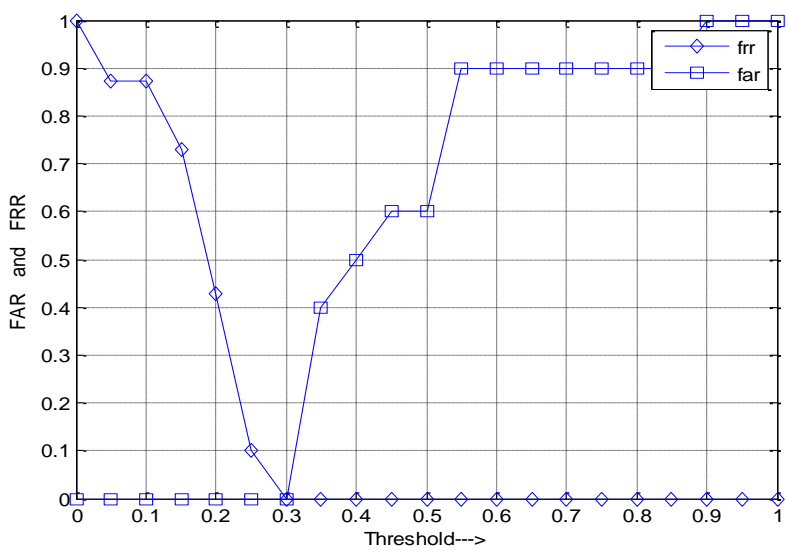

Fig.11: FRR and FAR v/s Threshold for proposed technique

Table 2: Comparision of CRR and EER for proposed and existing techniques

\begin{tabular}{|l|l|l|}
\hline Methods & CRR (\%) & EER (\%) \\
\hline Daugman [13] & 100 & 0.08 \\
\hline Boles and Boashash [14] & 92.64 & 8.13 \\
\hline L.Ma et al., [15] & 100 & 0.07 \\
\hline Chang et al., [16] & 98.79 & 1.29 \\
\hline Zhang et al., [17] & 100 & 0.46 \\
\hline Proposed Algorithm & 100 & 0.00 \\
\hline
\end{tabular}


The correct recognition rate and EER values of proposed IREF algorithm is compared with the existing algorithms viz. Daugman[13], Boles and Boashash[14], L.Ma et al., [15], Chang et al., [16], Zhang et al., [17]. It is observed that the proposed algorithm gives better results compared to existing algorithm. As per the Table 2, the proposed model gives 100\% CRR and zero EER compared to the existing algorithms.

\section{Conclusion}

Iris is unique part of a human body used to verify a person. In this paper Transform Domain Based Iris Recognition using EMD and FFT algorithm is proposed. The eye image is preprocessed to obtain ROI area from an iris part. The EMD and FFT are applied on ROI to extract features individually. The arithmetic summation is used to generate final features from individual features. The test iris features are compared with database iris features using ED. The proposed algorithm gives better CRR and EER values compared to existing algorithms. In future the algorithm may be tested with different transformations and fusion techniques.

\section{References}

[1] Ya-Ping Huang, Si-Wei Luo and En-Yi Chen, “An Efficient Iris Recognition System,” IEEE International Conference on Machine Learning and Cybernetics, vol. 1, pp. 450-454, November 2002.

[2] C. Tisse, L. Martin, L. Torres and M. Robert, "Person Identification Technique using Human Iris Recognition," IEEE International Conference on Vision Interface, vol. 4, pp. 33 - 36, 2004.

[3] J. Daugman, "How Iris Recognition Works," IEEE Transactions on Pattern Analysis and Machine Intelligence, vol. 14, pp. 21-30, 2004.

[4] Hui Zheng and Fei Su, “An Improved Iris Recognition System Based on Gabor Filters,” IEEE International Conference on Network Infrastructure and Digital Content, pp. 823-827, 2009.

[5] Lahouari Ghouti and Fares S. Al-Qunaieer, "Color Iris Recognition Using Quaternion Phase Correlation," IEEE Symposium on Bio-Inspired Learning and Intelligent Systems for Security, pp. 20-25, 2009.

[6] Ghassan J. Mohammad ,Hong BinKung, BinRong, Ann A. Al-Kazzaz and Maan Younis Abdullah, "A New Localization Method for Iris Recognition Based on Angular Integral Projection Function," IEEE International Workshop on Education Technology and Computer Science, pp. 316-320, 2009.

[7] Khin sint Kyaw, "Iris Recognition System using Statistical Features for Biometric Identification," IEEE International Conference on Electronic Computer Technology, pp. 554-556, 2009.

[8] H B Kekre, "Iris Recognition Using Vector Quantization," IEEE International Conference on Signal Acquisition and processing, pp 58-61, 2010

[9] Zhou Hu-Lin and Xie Mei, "Iris Biometric Processor Enhanced Module," IEEE International Conference on Computer Modeling and Simulation, pp. 259-262, 2010.

[10] C M Patil and Sudharshan Patilkulkarni, “An Approach of Iris Feature Extraction for Personal Identification," IEEE International Conference on Advances in Recent Technologies in Communication and Computing, pp.796-799, 2009.

[11] GU Hong-ying, Zhuang Yue-ting, PAN Yun-he, "An Iris Recognition Method Based on Multi- Orientation Features and Nonsymmetrical SVM," Journal of Zhejiang University, vol. 6, pp. 428-432, 2008.

[12] Makram Nabti and Ahmed Bouridane, "An Effective and Fast Iris Recognition System based on a Combined Multiscale Feature Extraction Technique,” Elsevier Science Inc. New York, NY, USA, pp. 868-879, 2008.

[13] J. Daugman's, "High Confidence Visual Recognition of Persons By A Test of Statistical Independence," IEEE Transactions on Pattern Analysis And Machine Intelligence, Vol. 15, No. 11, pp. 1148-1161, November, 1993.

[14] W.W. Boles and B. Boashash, "A Human Identification Technique using Images of the Iris and Wavelet Transform," IEEE Transactions on Signal Processing, vol. 46, no. 4, pp. 1185-1188, 1998.

[15] Li Ma, Tieniu Tan, Yunhong Wang and Dexin Zhang, "Efficient Iris Recognition by Characterizing Key Local Variations," IEEE Transactions on Image Processing, pp.739-750, 2004.

[16] Chien-ping Chang, Jen-Chun Lee, Yu Su and Te-Ming Tu "Using Empirical mode Decomposition for iris recognition," Elsevier Science Publishers B. V. Amsterdam, The Netherlands, Computer Standards and Interfaces Vol 31, Issue 4, pp 729-739, June, 2009

[17] Zhang Shunli, Han Min, Sun Weifeng and Yang Mingqiang "Iris Feature Extraction and Recognition Based On Empirical Mode Decomposition," IEEE International Conference on Fuzzy Systems and Knowledge Discovery, pp. 2633-2636, 2010

[18] Anna Linderhed "Image Empirical Mode Decomposition: A New Tool For Image Processing, "World Scientific Publishing Company Advances in Adaptive Data Analysis Vol. 1, No. 2, pp. 265-294, 2009

[19] Li Lin, Ji Hongbing "Signal feature extraction based on an improved EMD method," Elsevier publishers,Measurement,0263-2241 January 2009 